\title{
Assessment of COVID-19 Treatment containing both Hydroxychloroquine and Azithromycin: A Natural Clinical Trial
}

\author{
Hassan M Abbas ${ }^{1}$, Ali Azeez Al-Jumaili², Kawthar F Nassir ${ }^{3}$, Muhammed Waheeb \\ Al-Obaidy $^{4}$, Adnan Mohammed Al Jubouri ${ }^{4}$, Basim Dhawi Dakhil ${ }^{3}$, Mohammed Mahir \\ Abdulelah $^{3}$, and Qutaiba Ahmed Al Khames ${ }^{5}$ \\ ${ }^{1}$ Iraq Ministry of Health \\ ${ }^{2}$ University of Baghdad Bab Al-Moadham Campus College of Pharmacy \\ ${ }^{3}$ Baghdad Medical City \\ ${ }^{4}$ University of Baghdad Bab al-Moadham Campus College of Medicine \\ ${ }^{5}$ Philadelphia University Faculty of Pharmacy
}

June 26, 2020

\begin{abstract}
The goal of this study was to assess the clinical effectiveness and safety profile of the COVID-19 treatment protocol (containing both hydroxychloroquine (HCQ) and azithromycin) in an Iraqi specialized hospital. Methods: This prospective study used a pre- and post-intervention design without a comparison group. The intervention was routine Ministry of Health (MOH) approved management of COVID-19 for all patients. The study was conducted in a public healthcare setting in Baghdad, Iraq from March 1st to May 25, 2020. The study outcome measures included the changes in clinical and biochemical parameters during the hospitalization period. Paired t-test and Chi-square test were used to compare the measures of vital signs, lab tests and symptoms before and after treatment. Results: The study included 161 patients who were admitted with positive RT-PCR and clinical symptoms of COVID-19. In terms of severity, 53 (32.9\%) patients had mild condition, 47 (29.2\%) had moderate condition, $35(21.7 \%)$ had severe condition, and 26 (16.1\%) had critical condition. Most patients (84.5\%) recovered and were discharged without symptoms after testing negative with RT-PCR, while 11 (6.8\%) patients died during the study period. The signs and symptoms of COVID-19 were reduced significantly in response to therapy regimen containing HCQ and azithromycin. The most common reported side effects were stomach pain, hypoglycemia, dizziness, itching, skin rash, QT prolongation, arrhythmia, and conjunctivitis. Conclusions: This natural trial showed that COVID-19 regimen containing both HCQ and azithromycin can be helpful to promote recovery of most patients and reduced their signs and symptoms significantly. It also shows some manageable side effects mostly those related to heart rhythm. In the absence of FDA-approved medications to treat COVID-19, the repurposing of HCQ and azithromycin to control the disease signs and symptoms can be useful.
\end{abstract}

\section{What is already known about this topic?}

There is a controversy about using hydroxychloroquine and azithromycin to treat COVID-19 due to uncertainly of their effectiveness and safety.

\section{What does this article add?}

- The experience with Iraqi protocol to treat COVID-19 can help to answer some questions about the effectiveness and safety of this therapy combination which can offer some support to the international community in facing such vicious pandemic.

- This natural trial showed that COVID-19 regimen containing both HCQ and azithromycin can be helpful to promote recovery of most patients and reduced their signs and symptoms significantly. 
The trial was not registered because it is a natural trial (quasi experiment) which was conducted according the Iraqi Ministry of Health protocol by a regular healthcare team who is planning to share its experience with the international scientific community.

\section{Introduction}

Coronavirus disease 2019 (COVID-19) is a respiratory tract infection caused by a newly emergent coronavirus that was first recognized in Wuhan, China, in December $2019^{1}$. Genetic sequencing of the virus suggests that it is a beta-corona virus closely linked to the SARS virus ${ }^{2}$. While most people with COVID-19 develop only mild or uncomplicated illness, approximately $14 \%$ develop severe disease that requires hospitalization and oxygen support, and $5 \%$ require admission to an intensive care unit ${ }^{3}$. In severe cases, COVID-19 can be complicated by acute respiratory distress syndrome (ARDS), sepsis and septic shock, multiorgan failure, including acute kidney injury and cardiac injury ${ }^{3}$. Older age and co-existing diseases have been reported as risk factors for death ${ }^{4}$. On March 12, the WHO officially declared the COVID-19 outbreak a pandemic ${ }^{5}$. As of June 24, 2020, COVID-19 was confirmed in 188 countries with a total confirmed cases of 9,295,365 and more than 478,289 deaths ${ }^{6}$. Iraq had 36,702 confirmed cases, 16,814 recovered cases and 1,330 deaths associated with COVID-19 pandemic as of June 24, $2020^{6}$.

The pandemic of COVID-19 is caused by the novel severe acute respiratory syndrome coronavirus-2 (SARSCoV-2) and presents a challenge in regard to identifying effective drugs for prevention and treatment. Up to the time of writing this manuscript, there were no medications approved by the U.S. Food and Drug Administration (FDA) to treat COVID-197. However, the FDA recently created a new emergency program, the Coronavirus Treatment Acceleration Program (CTAP), aimed at speeding up research for the development of COVID-19 treatments ${ }^{8},{ }^{9}$. As of May 11, 2020, more than 144 clinical trials had been launched to test COVID-19 treatments, including some drug repurposing or repositioning ${ }^{8}(9)$. Researchers are also testing medications typically used to treat other conditions to see if they are also effective in treating COVID-19. For example, hydroxychloroquine (HCQ) and chloroquine are two medications that have been used for many decades to treat malaria and autoimmune conditions like rheumatoid arthritis and lupus ${ }^{10}$, and azithromycin is an antibiotic commonly used to treat bacterial infections such as bronchitis and pneumonia. They have been shown to have some in vitro activity against viruses like influenza $\mathrm{A}$ and $\mathrm{Zika}^{11}$.

We are ethically committed to share the experience of Iraqi healthcare providers with COVID-19 treatment with the scientific community to help answer some questions about the effectiveness and safety of HCQ and azithromycin containing regimen. The Iraqi Scientific Committee at the Ministry of Health (MOH) adopted a treatment protocol on March 1st, 2020 several days after the detection of the first COVID-19 case in Iraq on February 24, 2020 ( Table 1) ${ }^{12}$. The goal of this study was to assess the clinical effectiveness and safety profile of the current COVID-19 treatment protocol (containing both HCQ and azithromycin) in an Iraqi specialized hospital.

\section{Methods}

\section{Study Designs, duration and setting}

This prospective study used a pre- and post-intervention design. The intervention was the routine MOH approved management of COVID-19 for all patients. In other words, this was a natural clinical trial (quasi experiment) with no comparison group. The study was conducted in a public healthcare center, Al-Shifaa Center for COVID-19 pandemic treatment, Medical City, Baghdad, Iraq. The study included all adult patients with a confirmed COVID-19 infection that were admitted to this hospital over 85 days (from March 1st to May 25, 2020). They were defined as confirmed cases of coronavirus COVID-19 after the result of nasopharyngeal sample using Reverse transcription- polymerase chain reaction (RT-PCR) comes positive.

Exclusion criteria included known allergy to hydroxychloroquine (HCQ) or chloroquine, any contraindications to HCQ or chloroquine and azithromycin including retinopathy and prolonged QT, patients with severely reduced in left ventricular (LV) function, severely reduced in renal function and G6PD deficiency in addition to pregnant and lactating women. 


\section{Follow-up protocol and measures}

The research team (clinical pharmacist and three specialist physicians) was part of the medical team in the center. They prospectively collected patient medical information from patients, patient records and attending physicians. The information included demographics, disease and treatment characteristics and clinical outcome. Patient's clinical conditions and routine biochemical tests were assessed at baseline and then every day to evaluate the effect of therapy protocol.

\section{Study outcomes:}

The study outcome measures were assessed at baseline (at admission) and daily after hospitalization (postintervention) until discharge of the recovered patient or death. The study outcome measures included the changes in clinical and biochemical parameters during hospitalization period such as disappearance of clinical symptoms, virologic clearance and occurrence of side effects. The routine assessment of vital signs was conducted three times daily. Virologic clearance was measured using real time PCR which was retested at day 6 post hospitalization. Complete recovery was defined as two negative RT-PCR tests (which were usually done on days $6^{\text {th }}$ and $7^{\text {th }}$ of hospitalization). Fourteen days after hospital discharge, recovered patients were retested in outpatient clinics using RT-PCR.

Other outcome measures included the time to clinical recovery (TTCR) and final clinical prognosis (recovery vs death). The TTCR is defined as the time to disappearance of clinical symptoms (e.g. cough and shortness of breath, SOB) and a normal body temperature and respiratory rate for more than 72 hours.

\section{Study patients}

Study participants with confirmed cases of COVID-19 were transferred to the study specialized center after having a positive RT-PCR test at one of Medical City Hospitals. After admission, COVID-19 patients were classified according to clinical evaluation to mild cases (no pneumonia on a CT scan), moderate cases (pneumonia on a CT scan), severe cases (respiratory rate [?] 30 breaths /min, oxygen saturation [?] 93\% or patients with pneumonia on a CT scan) and critical cases (respiratory failure/need mechanical ventilation). All patients were treated according to the $\mathrm{MOH}$ treatment protocol which relies on patient severity status (Table 1).

To assess radiological changes, a chest CT scan was conducted before starting the treatment (day 0 ) and after 5 days of treatment (day 6). Pulmonary status was classified into three levels: Exacerbated, unchanged, and improved. Medication safety was also assessed by monitoring adverse drug reactions (ADRs), vital signs and lab tests. Routine lab tests during treatment period included liver function tests, renal function tests and fasting blood sugar. Liver function tests included alanine aminotransferase (ALT), aspartate aminotransferase (AST), alkaline phosphatase (ALP) and total bilirubin. Renal function was assessed using serum urea and creatinine (s.cr). The WHO-UPPSALA causality assessment criteria was used to determine the causality of adverse drug reactions (ADRs) of hydroxychloroquine and azithromycin ${ }^{13}$.

All data were recorded in the patient medical record. Consent was obtained from the patients before giving the therapy regimen. The researchers de-identified patient names and addresses to keep patient confidentiality. The study was approved by the Higher Ethical Committee at Medical City Hospitals.

\section{Statistical Analysis:}

Statistical Package for the Social Sciences 22 (SPSS, Chicago, IL, USA) was used to analyze the data. For descriptive analysis, categorical variables were presented as frequencies and percentages, while continuous variables were described using mean and standard deviation. A paired t-test was used to compare the means of vital signs and lab tests before and after treatment. The Chi-square test was used to compare categorical variables (presence of fever and cough) before and treatment. A p-value $<0.05$ was considered to be statistically significant.

\section{Results}


The study included 161 patients [52 (32.3\%) women, $109(67.7 \%)$ men] who were admitted with positive RT-PCR and clinical symptoms of COVID-19 (Table 2). The participants' mean age was $44.3( \pm 17.0)$ years and average bodyweight was $81.3( \pm 9.6)$. The disease was prevalent among the middle age group (41-50 years). One-fifth of patients (19.3\%) acquired the infection when they were visiting Iran. Approximately two-thirds $(63.4 \%)$ of patients were infected by contact with a family member while 17 (10.6\%) patients obtained COVID-19 from an unknown person. Some patients were healthcare providers/staff $(6.8 \%)$ who were infected due to their work in hospitals. In terms of severity, $53(32.9 \%)$ patients had mild condition, $47(29.2 \%)$ had moderate condition, $35(21.7 \%)$ had severe condition, and $26(16.1 \%)$ had critical condition. Approximately, one-quarter $(23.6 \%)$ of the patients were admitted to the respiratory care unit (RCU) (Table $2)$. All the 11 deceased patients were admitted in critical condition and had co-existing diseases.

The most common symptoms of COVID-19 patients at admission were cough (64.6\%), headache (56.5\%), shortness of breath (SOB) (47.2\%), sore throat (41.0\%), diarrhea (36.6\%), exertional dyspnea (32.9\%), abdominal pain $(31.1 \%)$, tiredness $(31.1 \%)$, loss of appetite $(29.8 \%)$, vomiting $(23.6 \%)$, chest tightness $(15.5 \%)$ (Table 3).

Most patients $(84.5 \%)$ recovered and were discharged without symptoms after testing negative RT-PCR. However, 14 (8.7\%) patients were discharged with mild symptoms (mild cough and/or exertional dyspnea). All recovered patients were required to be retested with RT-PCR 14 days after their hospital discharge.

On the other hand, 11 (6.8\%) patients died during the study period. Eight of them died before completing the five-day treatment course (six died within the first 24 hours and two died after 24 hours), while three died after one week of treatment in the intensive care unit (ICU) (Table 4). The deceased patients were admitted to the hospital in critical condition.

The average period of patient hospitalization was 12.9 ( \pm 5.5$)$ days. However, the duration of treatment was up to 14 days (for patients with pneumonia). The standard COVID-19 regimen which included hydroxychloroquine $(\mathrm{HCQ})$ and azithromycin had a significant positive effect $(\mathrm{P}$-value $<0.05)$ on patient body temperature, cough, $\mathrm{SOB}$, respiratory rate $(\mathrm{RR})$, pulse rate $(\mathrm{PR})$, and fasting blood sugar (Table 5). In other words, those signs and symptoms of COVID-19 patients were reduced significantly after using therapy regimen containing HCQ and azithromycin. However, there were non-significant effects of treatment (P-value [?]0.05) on systolic blood pressure (SBP), diastolic blood pressure (DBP), AST, ALT, ALP, total bilirubin, serum urea, and serum creatinine (Figures 1 \& 2).

In terms of adverse drug reactions (ADRs), the most common reported ones were stomach pain/cramps, hypoglycemia, headaches, mood changes, dizziness, itching, diarrhea, muscle weakness, nausea, skin rash, vomiting, QT prolongation, arrhythmia, and conjunctivitis (Figure 3). Most ADRs were possible to be caused by HCQ and/or azithromycin. It means they also can be caused by the disease (COVID-19). On the other hand, three ADRs including arrhythmia, QT prolongation and conjunctivitis were probably caused by the two medications (Table 6). In other words, they unlikely to be attributed to COVID-19 disease. The electrocardiograph (ECG) of six $(3.7 \%)$ patients recorded QTc prolongation, but not more than $450 \mathrm{~ms}$. Additionally, arrhythmia was reported in three $(1.9 \%)$ other patients (Table 6 ). The medical team stopped the HCQ and azithromycin treatment for three patients who experienced arrhythmia. Then electrolyte levels (calcium and potassium) were measured and corrected to treat the arrhythmia. However, this treatment stoppage occurred after completing the first five days of the therapy course.

\section{Discussion:}

The pandemic of coronavirus disease 2019 (COVID-19) caused by the SARS-CoV-2 presents a challenge in terms of identifying effective drugs for prevention and treatment ${ }^{14}$. HCQ has a long history as safe and inexpensive drug for malaria and autoimmune diseases despite some eye and cardiac side effects ${ }^{15}$.

During the study period (from March 1 to May 25, 2020) and before the exponential increase in COVID-19 cases in Iraq ( $>1000$ cases/day) which started on June 5, 2020, admission to one of the MOH quarantine facilities was required by law for confirmed cases. Thus, all patients who tested positive were treated in 
government COVID-19 specialized facilities. The study center was one of 22 specialized COVID-19 centers across the country. The number of participants in this study represents 3.5\% of the total Iraqi COVID-19 cases during the study period. The therapy regimen was the same across all 22 specialized COVID-19 in Iraq as it was implemented by the MOH. According to the MOH therapy protocol, HCQ and azithromycin should be given for all COVID-19 cases and other anti-viral agents/antibiotics can be added for severe and critical cases ${ }^{12}$. During the study period, the use of convalescent plasma to treat COVID-19 had not been started in the study setting.

In this study, the treatment regimen (containing both HCQ and azithromycin) appeared to help promote recovery in 150 COVID-19 patients (83.2\%) in the study center. Although $16 \%$ of patients were admitted in critical condition and $26 \%$ were admitted to the RCU, the mortality rate was only $6.8 \%$. It is worth mentioning that most deceased patients were admitted in critical condition and died before receiving full course of treatment. The same MOH therapy regimen facilitate recovery in 2,811 cases out of 4,632 COVID19 patients in Iraq during the study period, and only 163 deaths $(3.52 \%)$ were reported ${ }^{6}$. This high recovery rate was probably due to the effectiveness of the treatment protocol and close disease monitoring by healthcare providers. They work hard despite several challenges facing Iraqi healthcare system including a shortage in number of quarantine facilities, a shortage in personal protective equipment (PPE) and limited RT-PCR testing ${ }^{16}$.

The average of hospitalization period was 12.9 (+-5.5 days) given there was small number of admissions per day during the study period and no high demand for hospital beds. This duration may reflect the fact that about two-third of admitted patients developed pneumonia and needed time to recover. On the other hand, all those who tested negative with RT-PCR on days 6 and 7 after admission were discharged on day 8. The results of our study were comparable to a French clinical trial to evaluate the role of HCQ and Azithromycin on respiratory viral loads in patients with confirmed COVID-19 ${ }^{17}$. The study found a significant reduction of the viral carriage in HCQ/azithromycin group compared to control group at day 6 post-inclusion. The study reported 100\% viral clearance in nasopharyngeal swabs with combination of HCQ and azithromycin compared to $57.1 \%$ in HCQ alone group and $12.5 \%$ in patients who did not receive HCQ $(\mathrm{p}<0.001)^{17}$. Chloroquine and HCQ appear to block viral entry into cells by inhibiting glycosylation of host receptors, proteolytic processing, and endosomal acidification ${ }^{1819}$. These agents also have immunomodulatory effects through attenuation of cytokine production and inhibition of autophagy and lysosomal activities in host cells ${ }^{19},{ }^{20}$. HCQ has in-vitro activity against SARS-CoV-2 with half-maximal effective concentration (EC50) of $6.14 \mu \mathrm{M}$ which is lower than that of chloroquine $(\mathrm{EC} 50=23.90 \mu \mathrm{M})$ after 24 hours of growth ${ }^{18}$.

On the other hands, some manageable side effects of HCQ were reported during treatment period. These side effects may disappear during treatment as the body adjusts to the medicine ${ }^{21}$. The reported side effects included stomach pain/cramps, hypoglycemia, headaches, mood changes, dizziness, itching, diarrhea, muscle weakness, nausea, skin rash, and vomiting. Those are common HCQ side effects and appear to be dose-dependent and most often occur with loading doses of $800 \mathrm{mg}$ or higher ${ }^{22}$. In this study, we used an HCQ maintenance dose of 400 mag daily for short-term course which is relatively safe since the recommended daily dose was not exceeded. The most severe and life-threatening complications from use of hydroxychloroquine include QT interval prolongation and the resultant risk of ventricular arrhythmias 23. In our study, the electrocardiogram (ECG) monitoring showed that six patients developed QT interval prolongation, but not more than $450 \mathrm{~ms}$ which did not require medical intervention. In contrast, three patients developed arrhythmia and needed medical intervention which consisted of stopping the treatment and normalizing the blood electrolytes (potassium and calcium). The incidence of QT interval prolongation with using chloroquine and hydroxychloroquine is highly dependent on baseline ECG findings, with risk exacerbated using concomitant QT-interval prolonging medications ${ }^{24}$. The combination of azithromycin with HCQ frequently prolongs the QT interval in a clinically significant manner, increasing over time and requiring additional monitoring ${ }^{25}$.

Chinese Centre for Disease Control and Prevention includes chloroquine in the treatment guideline for COVID-19 patients which gave high recovery rate $(94.5 \%)^{26}$. A recent study relying on a multinational 
registry analysis which was published on May 22, 2020 found no evidence of hydroxychloroquine effectiveness to treat COVID-1927. However, this study was retracted after some expressed concerns about its findings. On March 28, 2020, the U.S. FDA issued Emergency Use Authorizations (EUA) that allowed the use of hydroxychloroquine to treat COVID-19. On June 15, 2020, the FDA revoked this EUA due to the absence of scientific evidence ${ }^{28}$. This controversy about using hydroxychloroquine to treat COVID-19 is due to the absence of findings based on well-designed randomized controlled trials (RCTs). Although there is a debate about the effectiveness and safety of using hydroxychloroquine, the new Iraqi guideline (on June 1, 2020) still includes HCQ to treat mild and moderate cases of COVID-19 since it has been showing promising results and due to the absence of superior alternatives, in addition to its affordability and availability.

This study had some limitations. It was conducted in a single center without a comparison group. Single center findings may not be generalizable. However, a universal therapy regimen has been implemented by the health top authority across all country healthcare settings. Lack of a comparison group can limit the determination of causality between the therapy and the cure or good prognosis. Additionally, some disease-induced symptoms overlap with ADRs during treatment period.

\section{Conclusions}

This natural trial showed that COVID-19 regimen containing both HCQ and azithromycin is helpful in treating the majority of patients and reduced their signs and symptoms significantly. It also causes some manageable side effects mostly those related to heart rhythm. In the absence of FDA-approved medications to treat COVID-19, the repurposing of HCQ and azithromycin to control the disease signs and symptoms is potentially useful. The study showed that using this therapy combination (HCQ and azithromycin) is promising and can fill the gap until more effective treatments are found. Future randomized controlled trial can give more definite answers about the effectiveness and safety of such therapy protocol.

Table 1: Iraqi COVID-19 treatment protocol (13)

\begin{tabular}{ll}
\hline Therapy regimen & Case Severity \\
\hline Hydroxychloroquine PO (400mg BID first day then & Covid-19 patients without pneumonia \\
$200 \mathrm{mg}$ BID for 5 days), Azithromycin PO (500mg & \\
in the first day, then $250 \mathrm{mg}$ daily for 5 days $)$ & \\
Hydroxychloroquine PO (400mg BID first day then & Covid-19 patients with pneumonia in the ward \\
$200 \mathrm{mg}$ BID for 14 days), Azithromycin PO & \\
(500mg in the first day, then $250 \mathrm{mg}$ daily for 14 & \\
days), Tamiflu $75 \mathrm{mg}$ PO BID for 5 days & \\
Hydroxychloroquine PO (400mg BID first day then & Covid-19 patients with pneumonia in the ICU \\
$200 \mathrm{mg}$ BID for 14 days), Azithromycin PO & \\
(500mg in the first day, then $250 \mathrm{mg}$ daily for 14 & \\
days), Tamiflu $75 \mathrm{mg}$ PO BID for 5 days, Kaletra & \\
(Lopinavir $200 \mathrm{mg} /$ ritonavir $50 \mathrm{mg}$ ) BID for 5 & \\
days and antibiotic(s) accordingly &
\end{tabular}

"PO" means the medication is taken by mouth. ICU=intensive care unit.

$\mathrm{BID}=$ twice a day.

Table 2: Demographic and disease characteristics for patients

\begin{tabular}{llll}
\hline Characteristics & Characteristics & Characteristics & N $(\%)$ \\
\hline Age (years) & Age (years) & $18-30$ & $35(21.73)$ \\
& & $31-40$ & $30(18.63)$ \\
& & $41-50$ & $41(25.46)$
\end{tabular}




\begin{tabular}{|c|c|c|c|}
\hline Characteristics & Characteristics & Characteristics & $\mathrm{N}(\%)$ \\
\hline & & $51-60$ & $31(19.25)$ \\
\hline & & $61-80$ & $24(14.90)$ \\
\hline & & Total & $161(100)$ \\
\hline \multirow[t]{2}{*}{ Gender } & Female & Female & $52(32.3)$ \\
\hline & Male & Male & $109(67.7)$ \\
\hline $\begin{array}{l}\text { Exposure and } \\
\text { transmission }\end{array}$ & $\begin{array}{l}\text { Exposure and } \\
\text { transmission }\end{array}$ & $\begin{array}{l}\text { Exposure and } \\
\text { transmission }\end{array}$ & $\begin{array}{l}\text { Exposure and } \\
\text { transmission }\end{array}$ \\
\hline $\begin{array}{l}\text { Travelers returning } \\
\text { from affected areas }\end{array}$ & $\begin{array}{l}\text { Travelers returning } \\
\text { from affected areas }\end{array}$ & $\begin{array}{l}\text { Travelers returning } \\
\text { from affected areas }\end{array}$ & $32(19.9)$ \\
\hline $\begin{array}{l}\text { Exposure direct } \\
\text { contact with patient } \\
\text { (most of them same } \\
\text { family) }\end{array}$ & $\begin{array}{l}\text { Exposure direct } \\
\text { contact with patient } \\
\text { (most of them same } \\
\text { family) }\end{array}$ & $\begin{array}{l}\text { Exposure direct } \\
\text { contact with patient } \\
\text { (most of them same } \\
\text { family) }\end{array}$ & $102(63.35)$ \\
\hline $\begin{array}{l}\text { Contact with unknown } \\
\text { person }\end{array}$ & $\begin{array}{l}\text { Contact with unknown } \\
\text { person }\end{array}$ & $\begin{array}{l}\text { Contact with unknown } \\
\text { person }\end{array}$ & $17(10.55)$ \\
\hline $\begin{array}{l}\text { Healthcare provider/ } \\
\text { staff }\end{array}$ & $\begin{array}{l}\text { Healthcare provider/ } \\
\text { staff }\end{array}$ & $\begin{array}{l}\text { Healthcare provider/ } \\
\text { staff }\end{array}$ & $11(6.83)$ \\
\hline Co-existing diseases & Co-existing diseases & Co-existing diseases & Co-existing diseases \\
\hline Hypertension & Hypertension & Hypertension & $38(23.60)$ \\
\hline $\begin{array}{l}\text { Diabetes with A1c > } \\
7.6 \%\end{array}$ & $\begin{array}{l}\text { Diabetes with A1c > } \\
7.6 \%\end{array}$ & $\begin{array}{l}\text { Diabetes with A1c > } \\
7.6 \%\end{array}$ & $37(22.98)$ \\
\hline Ischemic heart diseases & Ischemic heart diseases & Ischemic heart diseases & $8(4.96)$ \\
\hline Chronic kidney diseases & Chronic kidney diseases & Chronic kidney diseases & $4(2.48)$ \\
\hline Asthma & Asthma & Asthma & $8(4.96)$ \\
\hline $\begin{array}{l}\text { History of transplant or } \\
\text { other Immunosuppression }\end{array}$ & $\begin{array}{l}\text { History of transplant or } \\
\text { other Immunosuppression }\end{array}$ & $\begin{array}{l}\text { History of transplant or } \\
\text { other Immunosuppression }\end{array}$ & $2(1.24)$ \\
\hline Hepatitis B, C & Hepatitis B, C & Hepatitis B, C & $2(1.24)$ \\
\hline $\begin{array}{l}\text { Systemic lupus } \\
\text { erythematosus (SLE) }\end{array}$ & $\begin{array}{l}\text { Systemic lupus } \\
\text { erythematosus (SLE) }\end{array}$ & $\begin{array}{l}\text { Systemic lupus } \\
\text { erythematosus (SLE) }\end{array}$ & $1(0.62)$ \\
\hline Pulmonary & Pulmonary & Pulmonary & $1(0.62)$ \\
\hline hypertension & hypertension & hypertension & \\
\hline $\begin{array}{l}\text { Lymphocytopenia at } \\
\text { admission }\end{array}$ & $\begin{array}{l}\text { Lymphocytopenia at } \\
\text { admission }\end{array}$ & $\begin{array}{l}\text { Lymphocytopenia at } \\
\text { admission }\end{array}$ & $30(18.63)$ \\
\hline \multirow[t]{4}{*}{ Condition severity } & Mild & Mild & $53(32.91)$ \\
\hline & Moderate & Moderate & $47(29.19)$ \\
\hline & Sever & Sever & $35(21.73)$ \\
\hline & Critical & Critical & $26(16.14)$ \\
\hline $\begin{array}{l}\text { Needed respiratory } \\
\text { care unit (RCU) }\end{array}$ & $\begin{array}{l}\text { Needed respiratory } \\
\text { care unit (RCU) }\end{array}$ & $\begin{array}{l}\text { Needed respiratory } \\
\text { care unit (RCU) }\end{array}$ & $38(23.60)$ \\
\hline Needed oxygen therapy & Needed oxygen therapy & Needed oxygen therapy & $61(37.88)$ \\
\hline Characteristic & Characteristic & Characteristic & Mean (ST Dev) \\
\hline Age (years) & Age (years) & Age (years) & $44.60 \pm 16.44$ \\
\hline Body weight (kg) & Body weight (kg) & Body weight (kg) & $81.79 \pm 9.64$ \\
\hline $\begin{array}{l}\text { Duration of starting } \\
\text { symptoms before } \\
\text { admission (days) }\end{array}$ & $\begin{array}{l}\text { Duration of starting } \\
\text { symptoms before } \\
\text { admission (days) }\end{array}$ & $\begin{array}{l}\text { Duration of starting } \\
\text { symptoms before } \\
\text { admission (days) }\end{array}$ & $5.24 \pm 2.57$ \\
\hline
\end{tabular}

Table 3: COVID-19 patient symptoms at admission 


\begin{tabular}{lll}
\hline Symptom & Frequency (N) & Percent (\%) \\
\hline Cough & 104 & 64.6 \\
Headache & 91 & 56.5 \\
Shortness of breath (SOB) & 76 & 47.2 \\
Sore throat & 66 & 41.0 \\
Diarrhea & 59 & 36.6 \\
Exertional dyspnea & 53 & 32.9 \\
Abdominal pain & 50 & 31.1 \\
Tiredness & 50 & 31.1 \\
Loss of appetite & 48 & 29.8 \\
Vomiting & 38 & 23.6 \\
Chest tightness & 25 & 15.5 \\
Sneezing & 7 & 4.3 \\
Runny nose & 2 & 1.2 \\
Loss of taste/smell & 1 & 0.6 \\
Total & 161 & 100.0 \\
\hline
\end{tabular}

Table 4. The clinical outcomes of COVID-19 patients

\begin{tabular}{llr}
\hline Patient category & Patient category & N (\%) \\
\hline Total number of admitted patients & Total number of admitted patients & $161(100$ \\
Recovered patients & Recovered patients & $136(84$. \\
Discharged (recovered) patients with mild symptoms & Negative RT-PCR with mild cough or exertional dyspnea & $14(8.69$ \\
Death & Within 24 hours & $6(3.72 \%$ \\
& After 24 hours & $2(1.24 \%$ \\
& During the treatment & $3(1.86 \%$ \\
Hospitalization days for recovered patients & Total death & $11(6.83$ \\
& Mean \pm SD & $12.88 \pm$ \\
\hline
\end{tabular}

$\mathrm{SOB}=$ shortness of breath

Table 5: The effect of treatment on clinical condition and biochemical parameters of patients with COVID -19

\begin{tabular}{llll}
\hline Parameters & Before treatment & After treatment & P value \\
\hline Symptoms & $\mathrm{N}(\%)$ & $\mathrm{N}(\%)$ & \\
Cough & $104(64.60 \%)$ & $14(8.70 \%)$ & $.0000^{*}$ \\
SOB & $76(47.21 \%)$ & $11(6.83 \%)$ & $.0000^{*}$ \\
Fever & $38.25 \pm 0.64$ & $36.53 \pm 0.39$ & $.0000^{*}$ \\
Vital signs & Mean $\pm \mathrm{SD}$ & Mean $\pm \mathrm{SD}$ & \\
RR & $23.43 \pm 5.55$ & $19.66 \pm 2.32$ & $.0000^{*}$ \\
SBP & $129.27 \pm 16.68$ & $126.57 \pm 14.88$ & .1260 \\
DBP & $83.77 \pm 10.64$ & $84.76 \pm 10.46$ & .4015 \\
PR & $96.36 \pm 8.31$ & $78.21 \pm 3.05$ & $.0000^{*}$ \\
Lab tests & & & \\
FBS mmol/L & $9.61 \pm 5.65$ & $6.95 \pm 1.72$ & $.0000^{*}$ \\
WBCs *10 & $11.20 \pm 3.33$ & $6.67 \pm 1.97$ & $.0000^{*}$ \\
Hg g $\backslash \mathrm{dl}$ & $12.74 \pm 1.75$ & $12.70 \pm 1.54$ & .7969
\end{tabular}




\begin{tabular}{llll}
\hline Parameters & Before treatment & After treatment & P value \\
\hline S.Cr & $1.00 \pm 0.94$ & $0.95 \pm 0.69$ & .6451 \\
Urea & $26.04 \pm 34.77$ & $25.61 \pm 33.85$ & .9112 \\
AST & $25.32 \pm 13.16$ & $27.20 \pm 9.34$ & .1387 \\
ALT & $33.63 \pm 28.36$ & $34.80 \pm 20.15$ & .6925 \\
ALP & $106.47 \pm 71.16$ & $107.27 \pm 47.38$ & .9054 \\
Bilirubin & $0.73 \pm 0.70$ & $0.75 \pm 0.57$ & .7391 \\
\hline
\end{tabular}

*Significant p-value [?] 0.05 according to paired T-test or Chi-square. SOB=shortness of breath, $\mathrm{RR}=$ respiratory rate, $\mathrm{SBP}=$ systolic blood pressure, $\mathrm{DBP}=$ diastolic blood pressure, $\mathrm{PR}=$ pulse rate, FBS=fasting blood sugar, WBCs=white blood cells, $\mathrm{S} . \mathrm{Cr}=$ serum creatinine, AST=aspartate aminotransferase, ALT=alanine aminotransferase, ALP=alkaline phosphatase.

Table 6: The reported adverse reactions of HCQ \& azithromycin containing treatment on patients with COVID -19

\begin{tabular}{lll}
\hline Side effects & $\mathrm{N}(\%)$ & Causality term \\
\hline Stomach pain or cramps & $65(40.37 \%)$ & possible \\
Hypoglycemia & $60(42.86 \%)$ & possible \\
Headaches & $57(35.40 \%)$ & possible \\
Mood changes & $55(34.16)$ & unlikely \\
Dizziness & $50(31.06 \%$ & possible \\
Itching & $47(29.19 \%)$ & possible \\
Diarrhea & $45(27.95 \%)$ & possible \\
Muscle weakness & $41(25.47 \%)$ & possible \\
Nausea & $40(24.84 \%)$ & possible \\
Skin rash & $35(21.74 \%)$ & possible \\
Vomiting & $30(18.63 \%)$ & possible \\
QT prolongation & $6(3.73 \%)$ & probable \\
Conjunctivitis & $4(2.48 \%)$ & probable \\
Arrhythmia & $3(1.86 \%)$ & probable \\
\hline
\end{tabular}

Probable $=$ Unlikely to be attributed to disease or other drugs. Possible $=$ Could also be explained by disease or other drugs. Unlikely=Disease or other drugs provide plausible explanations ${ }^{13}$.

Figure 1: The effect of treatment on patient clinical condition (signs and symptoms)

Before therapy was measured at baseline (at admission). Vital sign measurements after therapy equal the average of the last three-day measurements. Frequency of patients with symptoms (cough/SOB) after therapy was measured on last day before discharge. $\mathrm{SOB}=$ shortness of breath, $\mathrm{RR}=$ respiratory rate, $\mathrm{SBP}=$ systolic blood pressure, $\mathrm{DBP}=$ diastolic blood pressure, $\mathrm{PR}=$ pulse rate.

Figure 2: The effect of treatment on FBS, WBCs, and $\mathrm{Hg}$ levels

FBS=fasting blood sugar; WBCs=white blood cells; $\mathrm{Hg}=$ hemoglobin.

Figure 3-The reported adverse reactions of treatment on patients with COVID -19

\section{References}

1. Guo YR, Cao QD, Hong ZS, et al. The origin, transmission and clinical therapies on coronavirus disease 2019 (COVID-19) outbreak- A n update on the status. Mil Med Res . 2020;7(1):1-10. doi:10.1186/s40779020-00240-0 
2. MacKenzie JS, Smith DW. COVID-19: A novel zoonotic disease caused by a coronavirus from China: What we know and what we don't.Microbiol Aust . 2020;41(1):45-50. doi:10.1071/MA20013

3. S. Tamblyn. Clinical Management of Patients with Moderate to Severe COVID-19 - Interim Guidance. Public Heal Agency Canada . 2020:1-20.

4. Zhou F, Yu T, Du R, et al. Clinical course and risk factors for mortality of adult inpatients with COVID-19 in Wuhan, China: a retrospective cohort study. Lancet . 2020;395(10229):1054-1062. doi:10.1016/S01406736(20)30566-3

5. Cucinotta D, Vanelli M. WHO declares COVID-19 a pandemic. Acta Biomed . 2020;91(1):157-160. doi:10.23750/abm.v91i1.9397

6. And $\mathrm{C}$ for SS, Engineering (CSSE) JHU (JHU). COVID-19 Dashboard. https://coronavirus.jhu.edu/map.html. Published 2020. Accessed June 24, 2020.

7. Ullah MA, Araf Y, Sarkar B, Moin AT, Reshad RAI, Rahman MH. Pathogenesis, Diagnosis and Possible Therapeutic Options for COVID-19. 2020;(April). doi:10.20944/PREPRINTS202004.0372.V1

8. FDA. Coronavirus Treatment Acceleration Program (CTAP)|. https://www.fda.gov/drugs/coronaviruscovid-19-drugs/coronavirus-treatment-acceleration-program-ctap. Published 2020. Accessed June 3, 2020.

9. Agranat ID and I. Chiral switches of chloroquine and hydroxychloroquine: potential drugs to treat COVID-19. Drug Discov Today . 2020. doi:https://doi.org/10.1016/j.drudis.2020.04.021

10. Gbinigie K, Frie K. Should chloroquine and hydroxychloroquine be used to treat COVID-19? A rapid review. BJGP Open . 2020:bjgpopen20X101069. doi:10.3399/bjgpopen20x101069

11. Damle B, Vourvahis M, Wang E, Leaney J, Corrigan B. Clinical Pharmacology Perspectives on the Antiviral Activity of Azithromycin and Use in COVID-19. Clin Pharmacol Ther . 2020;0(0):1-11. doi:10.1002/cpt.1857

12. Allawi JS, Abbas HM, Rasheed JI, et al. The first 40-days experience and clinical outcomes in the management of coronavirus covid-19 crisis. Single center preliminary study. J Fac Med Baghdad . 2019;61(3,4). doi:10.32007/J FAC MED BAGDAD.\%Y.613,4\%P

13. Center WHO-TUM. WHO Causality assessment. Good Pharmacovigil Pract Guid . 2009;(3):39.

14. Lai CC, Shih TP, Ko WC, Tang HJ, Hsueh PR. Severe acute respiratory syndrome coronavirus 2 (SARSCoV-2) and coronavirus disease-2019 (COVID-19): The epidemic and the challenges. Int $J$ Antimicrob Agents . 2020;55(3):105924. doi:10.1016/j.ijantimicag.2020.105924

15. Haładyj E, Sikora M, Felis-Giemza A, Olesinska M. Antimalarials - are they effective and safe in rheumatic diseases? Reumatologia . 2018;56(3):164-173. doi:10.5114/reum.2018.76904

16. Mikhael EM, Al-Jumaili AA. Can developing countries face novel coronavirus outbreak alone? The Iraqi situation. Public Heal Pract . 2020;1(March):100004. doi:10.1016/j.puhip.2020.100004

17. Gautret P, Lagier J-C, Parola P, et al. Hydroxychloroquine and azithromycin as a treatment of COVID-19: results of an open-label non-randomized clinical trial. Int J Antimicrob Agents . 2020:105949. doi:10.1016/j.ijantimicag.2020.105949

18. Al-Bari MAA. Targeting endosomal acidification by chloroquine analogs as a promising strategy for the treatment of emerging viral diseases. Pharmacol Res Perspect . 2017;5(1):1-13. doi:10.1002/prp2.293

19. Sanders JM, Monogue ML, Jodlowski TZ, Cutrell JB. Pharmacologic Treatments for Coronavirus Disease 2019 (COVID-19): A Review. JAMA - J Am Med Assoc . 2020;323(18). doi:10.1001/jama.2020.6019

20. Meyerowitz EA, Vannier AGL, Friesen MGN, et al. Rethinking the role of hydroxychloroquine in the treatment of COVID-19. FASEB J . 2020;34(5):6027-6037. doi:10.1096/fj.202000919 
21. Y Ikeda, R Rokutanda MO. THE SIDE EFFECT AND THE RETENTION RATE OF HYDROXYCHLOROQUINE IN JAPAN. St Luke's Int Hosp Tokyo, Japan . 2018;5((Suppl 1)):A1-A129.

22. Tétu P, Hamelin A, Lebrun-Vignes B, et al. [Prevalence of hydroxychloroquine-induced side-effects in dermatology patients: A retrospective survey of 102 patients]. Ann Dermatol Venereol . 2018;145(6-7):395404. doi:10.1016/j.annder.2018.03.168

23. O'Laughlin JP, Mehta PH, Wong BC. Life Threatening Severe QTc Prolongation in Patient with Systemic Lupus Erythematosus due to Hydroxychloroquine. Case Reports Cardiol . 2016;2016:1-4. doi:10.1155/2016/4626279

24. Gérard A, Romani S, Fresse A, et al. "Off-label" use of hydroxychloroquine, azithromycin, lopinavirritonavir and chloroquine in COVID-19: A survey of cardiac adverse drug reactions by the French Network of Pharmacovigilance Centers. Therapies . 2020. doi:10.1016/j.therap.2020.05.002

25. Archana Ramireddy MD1, Harpriya Chugh BS1, Kyndaron Reinier PhD1, Joseph 4 Ebinger MD1, Eunice Park BS2, Michael Thompson MS2, Eugenio Cingolani MD1, Susan 5 Cheng1, Eduardo Marban MD1, Christine M. Albert MD1 SSCM. Experience with Hydroxychloroquine and Azithromycin in the COVID-19 Pandemic: Implications for QT Interval Monitoring.medRxiv . 2020;21(1):1-9. doi:10.1016/j.solener.2019.02.027

26. Chinese Centre for Disease Control and Prevention. COVID-19 Prevention and Control .; 2020.

27. Mehra MR, Desai SS, Ruschitzka F, Patel AN. Hydroxychloroquine or chloroquine with or without a macrolide for treatment of COVID-19: a multinational registry analysis. Lancet . 2020. doi:10.1016/S01406736(20)31180-6

28. Coronavirus ( COVID-19 ) Update : FDA Revokes Emergency Use Authorization for Chloroquine and Hydroxychloroquine Inquiries. 2020:1-3.

\section{Hosted file}

Figures.docx available at https://authorea.com/users/337012/articles/462681-assessmentof-covid-19-treatment-containing-both-hydroxychloroquine-and-azithromycin-a-naturalclinical-trial 